\title{
Author Correction: New therapies for systemic lupus erythematosus - past imperfect, future tense
}

Grainne Murphy and David A. Isenberg

Nature Reviews Rheumatology (2019) https://doi.org/10.1038/s41584-019-0235-5

Published online 05 June 2019

The originally published article contained an error in the abstract. The sentence "Arguably, the success of $B$ cell depletion with rituximab in open-label clinical trials, the approval of belimumab (which blocks B cell-activating factor (BAFF)) for use in patients with lupus nephritis in the USA and in difficult-to-treat patients with SLE in the UK and the recognition that clinical trial design can be improved have given some cause for hope" has been corrected to "Arguably, the success of B cell depletion with rituximab in open-label studies and in patients with lupus nephritis in the USA and in difficult-to-treat patients with SLE in the UK, together with the approval of belimumab (which blocks B cell-activating factor (BAFF)) for use in patients with SLE and the recognition that clinical trial design can be improved, have given some cause for hope." This has been corrected in the HTML and PDF versions of the manuscript.

https://doi.org/10.1038/s41584-019-0262-2 I Published online 03 July 2019 